\title{
Accurate Robust Symmetry Estimation
}

\author{
Stephen Smith and Mark Jenkinson \\ FMRIB (Oxford Centre for Functional Magnetic Resonance Imaging of the Brain) \\ Department of Clinical Neurology, University of Oxford \\ John Radcliffe Hospital, Headington, Oxford OX3 9DU, UK \\ Tel: +44 1865222726 , Fax: +44 1865222717 \\ \{mark, steve\}@fmrib.ox.ac.uk ww. fmrib.ox.ac.uk
}

\begin{abstract}
There are various applications, both in medical and nonmedical image analysis, which require the automatic detection of the line (2D images) or plane (3D) of reflective symmetry of objects. There exist relatively simple methods of finding reflective symmetry when object images are complete (i.e., completely symmetric and perfectly segmented from image "background"). A much harder problem is finding the line or plane of symmetry when the object of interest contains asymmetries, and may not have well defined edges.

A major area of interest is brain image analysis; there are various reasons why one would want to be able to automatically, robustly and accurately find the (sagittal) mid-plane from a 3D brain image. Example applications include pre-alignment (or sanity checking) for standard registration methods, mid-plane finding as part of symmetric probabilistic anatomical map generation, and, in particular, symmetry-based analyses (e.g., for schizophrenia research). This paper describes EROS - Extraction of Robust Orientation using Symmetry, which has been developed to solve this problem. It has been shown to work with MRI (T1, T2, EPI), PET, SPECT and CT, using robust measures to give accurate results even with images containing large asymmetries.
\end{abstract}

Keywords: Symmetry detection, robust registration, mid-plane.

\section{Review}

Much of the existing work concentrates on defining symmetry, and developing low-level symmetry operators. For example, in the symmetry work reported by Di Gesù et al. (e.g., [1]), applied to such problems as face detection and astronomical image analysis, the algorithm is specifically tuned to the characteristics of these problems. The emphasis is on finding small symmetric features, rather than finding symmetries in large complex objects. Similarly, in [4], Reisfeld et al. find points of symmetry - there is no attempt at robust (larger) object symmetry detection.

Other work has looked for larger-scale symmetry, but often using constraints on the symmetries looked for. For example, in [9], vertical symmetry axes only are looked for in the context of road scene understanding for autonomous vehicle 
control. Sun et al. (e.g., [5]) use the extended gaussian image (EGI) to find different types of symmetries in 2D and 3D images. The EGI, however, is derived by looking at image gradient directions or the orientation of sections of image edges, and is therefore intrinsically noisy compared with looking for symmetry by direct point matching. The method also relies on finding principal axes first, and may therefore not be very robust to asymmetries in objects.

Many methods depend on first finding image edges before proceeding to detect symmetries. Clearly there are applications which cannot provide such well-defined features - for example, see the PET and SPECT images below. The approach reported in this paper does not require edges to be found; even when processing images with high bias field (see discussion below), only edge enhancement is used, not edge detection.

There are few published methods for finding symmetry in brain images. Minoshima et al. [3] present a method which detects the symmetry plane in PET images, using zero-crossing counting (stochastic sign change). Whilst it is shown to be robust to large image asymmetries, it is hard to see how the method could be extended to overcome even small bias fields in MRI images. Thirion et al. [6] reflect the image about a pre-assumed symmetry plane, and then use registration to align the original and reflected images. From the estimated transformation the symmetry plane can be deduced. This is an impressive approach, but depends on the registration being able to cope perfectly with significant asymmetries in the brain image in order to function correctly - at the moment it appears that success is probably limited to cases of restricted asymmetry. The method is also dependent on having a very rough initial idea of where the symmetry plane is.

\section{Method Detail}

The method used to robustly find the plane of symmetry is now briefly outlined, followed by a more detailed explanation of the reasoning behind the system, and its implementation.

\section{Preprocessing:}

The image histogram is used to find robust estimates of the image "minimum" (5\% into the cumulative histogram) and "maximum" (95\%). Next a rough brain/background threshold is defined using these values; it is assumed to be $\min +0.1(\max -\min )$. Now a rough estimate of the position of the centre of gravity of the brain in the image is made, and an "average" brain radius is estimated by using the brain/background threshold and counting the number of voxels above this threshold. This radius is used to control subsampling when a multi-scale search for the optimal symmetry plane is carried out.

\section{Symmetry search algorithm (3D case):}

The main algorithm for the search for the symmetry plane is:

- For all possible mid-plane orientations (i.e., all angles $\alpha, \beta$ )

- For all lines $l$ perpendicular to mid-plane given by these angles (i.e., a $2 \mathrm{D}$ grid of lines through the mid-plane)

- $\quad$ Find centre of symmetry $(s(x)$ is symmetry score at position $x$ along line find peak value, $s_{l}\left(x_{p}\right)$ for line $l$ ) 
- $\quad$ Add peak symmetry score into a total 1D array $\left(S\left(x_{p}\right)=S\left(x_{p}\right)+s_{l}\left(x_{p}\right)\right)$

- $\quad$ Find peak in total array $=$ score for these angles $\left(P_{\alpha, \beta}=M A X(S(x))\right)$ compare with peak found at other $(\alpha, \beta)$ so far.

See Figure 1 for example sets of lines perpendicular to a single choice of possible symmetry plane in each of three cases. (A single slice is shown, hence the symmetry plane is seen as a line.) The three cases are a "clean" image of a healthy brain, the "clean" brain with an incorrect choice of symmetry plane, and a brain with a large asymmetry superimposed. The position of the optimal centre of symmetry for each perpendicular line is shown. As a further clarification of how these positions are found, see Figure 2. In Figure 2 (left), the intensity profiles for three example lines in the "clean" and asymmetric cases are shown. Only one profile contains asymmetry - the final plot. In Figure 2 (right), the next processing stage for these plots is shown; the symmetry score at each possible centre of symmetry is given. Again, only the final plot shows asymmetry. Referring back to Figure 1, it is clear how the asymmetry has caused the marked centres of symmetry to shift.

Finally, all of the peak symmetry scores are combined across all perpendicular lines, into a single representative 1D array. Figure 3 (left) shows an example of this - the effect of the asymmetry is to give rise to non-aligned centres of symmetry in some of the perpendicular lines, causing the bulge on one side of the cumulative array. Figure 3 (right) shows these final arrays for the three cases shown earlier. When the mid-plane is incorrect, the peak symmetry scores will not all fall near the same $x$, so the total array will not contain as high a peak as when the plane is correct (the individual scores will be smaller as well, thus increasing the ability of the method to discriminate between correct and incorrect plane orientation).

Note that the lateral position of the symmetry plane, once the optimal angles have been selected, is immediately found from the position of the peak in the optimal cumulative 1D array. Clearly it would be easy to interpolate the values in this array if desired, to find a sub-voxel position.

Symmetry measure: The basic symmetry score used to find the centres of symmetry along each perpendicular line is:

$$
s(x)=\frac{\sum a b s\left(I_{x+i}+I_{x-i}\right)-\sum a b s\left(I_{x+i}-I_{x-i}\right)}{\sum a b s\left(I_{x+i}+I_{x-i}\right)+\sum a b s\left(I_{x+i}-I_{x-i}\right)}=\frac{e v e n-o d d}{\text { even }+ \text { odd }},
$$

where even is a measure of the "evenness" (unnormalised symmetry) and odd is a measure of the "oddness" (unnormalised asymmetry) of the intensity profile about $x$. This formulation provides normalization for intrinsic variations in intensity contrast and noise across the image. However, it incorrectly assigns too much weight to areas of the image which contain little signal. Thus a correction is made:

$$
s(x)=\frac{(\text { even }- \text { odd }) * l}{(\text { even }+ \text { odd })+g} .
$$

Correction $l$ is derived from local contrast (i.e., within the current perpendicular line), thus giving more weight to lines containing significant interest. Correction $g$ 


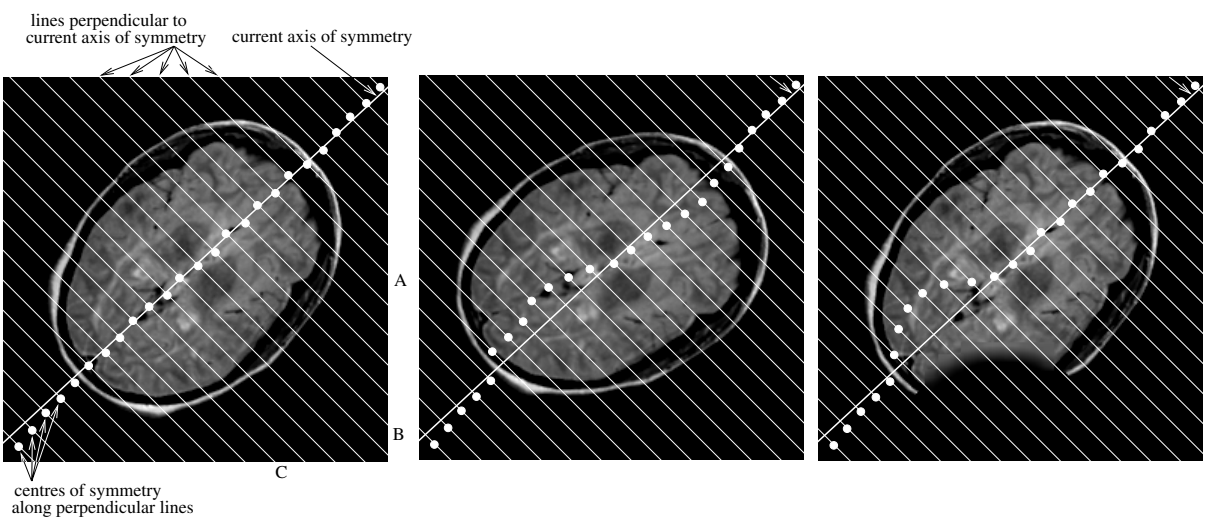

Fig. 1. Examples showing the set of lines perpendicular to proposed axes of symmetry in three cases with the position of centre of symmetry shown for each separate line.

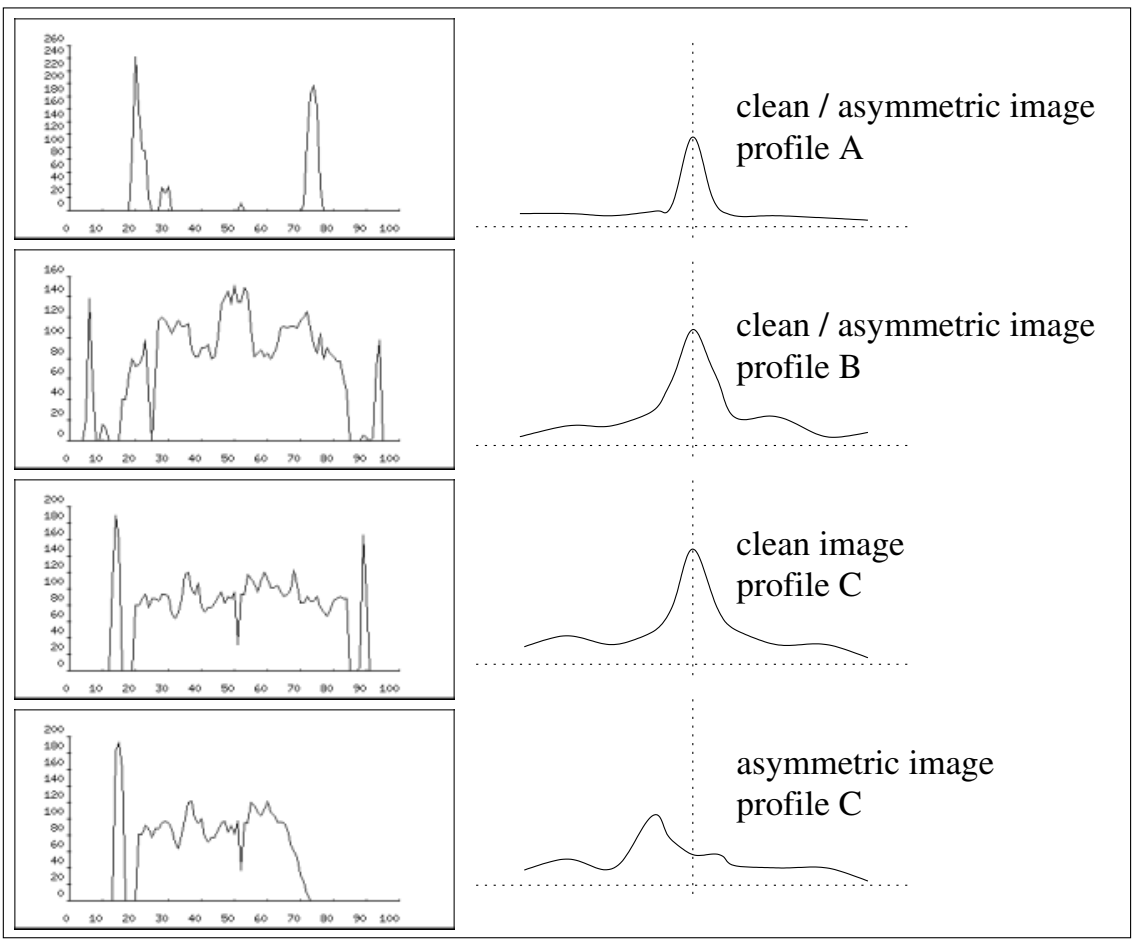

Fig. 2. Left: Intensity profiles along perpendicular lines A, B and C for Figure 1. Right: Symmetry score profiles along lines A, B and C; $x$ is the position (along the perpendicular line) of a proposed centre of symmetry, and $y$ is the symmetry score for that position. 


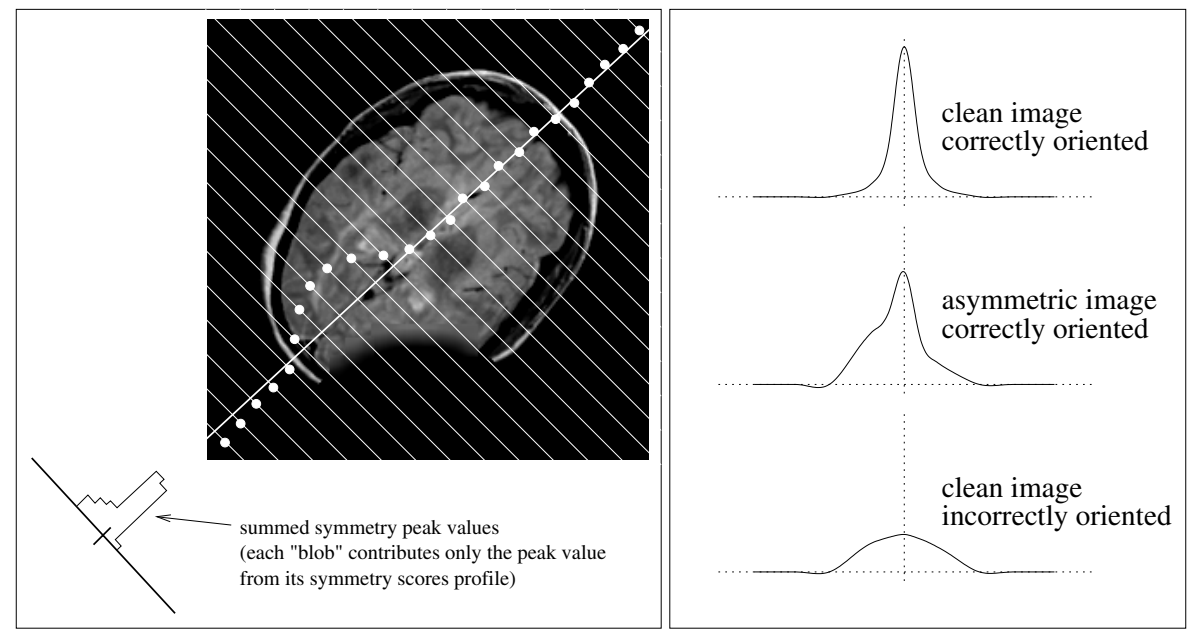

Fig. 3. Left: Example summed symmetry peak profile. Right: Example profiles for all images in Figure 1. As it is the position of the peak that matters, the asymmetry does not affect the estimate of the line of symmetry. The incorrectly oriented case gives a much lower peak than the correctly oriented case.

is a fraction of the global contrast, put here so that noise cannot have significant influence when there is little signal - a problem in background regions.

Robustness: EROS is robust to asymmetries (e.g., lesions or susceptibility artifacts) due to the method by which individual line symmetries are combined. The combination of all lines' symmetry positions into a single 1D array effectively produces a mode-like position statistic, although the robustness is increased even further than a true mode, where each peak would contribute an equal amount to the cumulative array. The contribution is the peak's value, not a constant, so that peaks derived from strong symmetries contribute more highly than those derived from weak symmetries.

A problem which has not been addressed thus far is that of a slowly varying additive or multiplicative field on top of the underlying image. This is common in MRI images, often termed bias field. EROS has been found to be robust to normal levels of bias field, i.e., still finds the correct plane of symmetry. However, with extreme bias field, simple symmetry does not exist - at optimal $x, I_{x+i}-I_{x-i}$ is not close to zero. In such cases, there is a very simple preprocessing stage which allows EROS to function correctly. By edge-enhancing the image (using a simple magnitude differential operator), the bias field is removed to first order. Successful mid-plane detection then results.

Computational efficiency measures: The calculations involved in robust symmetry detection are computationally expensive. A full search of all possible angles at full resolution is currently prohibitive. Therefore a multi-scale approach is taken - in practice, only two scales are used. Firstly, the image is smoothed 
and sub-sampled to give a smaller resulting image. Here the estimated brain size is used; it has been found empirically that a mean brain radius of 40 voxels in the subsampled image is the lowest resolution which still reliably gives a symmetry plane which is close to the "correct" solution. This solution is then used as the central point around which a restricted search is made at full (original) image resolution. The second measure to reduce computational cost is to only use a subset of all possible lines perpendicular to each tried symmetry plane. The spacing between these lines can be varied to speed up calculations. Currently, with these measures, EROS takes about 40 minutes to run on a typical MRI brain image, on a Silicon Graphics Origin 200.

Local detection: A particular application might either be more interested in the overall (global) optimal position for the plane of reflective symmetry (as has been assumed thus far), or, it may require more local detection, using only structures near the mid-plane to determine the exact position. The latter case is simple to accommodate - after the main algorithm, a final iteration can be applied, using only image points near the mid-plane in symmetry calculations, giving central detection instead of global.

\section{Results, Conclusions and Future Work}

Figure 4 shows example results from CT, SPECT and PET images respectively. These were 3D analyses - the central slice only is shown. The plane of symmetry thus shows as a line; the computed centre of gravity is also marked. Figure 5 (left) shows an MRI brain image with bad bias field artificially added. In order to allow EROS to succeed in finding the mid-plane, edge enhancement is carried out as a pre-processing step. Figure 6 (left) shows an MRI image with significant artificial asymmetries introduced. The simple non-robust solution is marked, as well as the robust one, which is correct. Figure 6 (right) shows symmetry detection on an image of the University of Ljubljani. The original image has been rotated and offset so that the symmetry line is not central in the image. The image was inverted so that the initial thresholding heuristics (easily alterable for different applications to brain analysis) would cause EROS to ignore the sky, and not the building!

EROS has not yet been found to fail to find the "correct" solution, except in investigations of how low, in resolution, the initial subsampled image can be. In terms of accuracy, the solution normally looks, by eye, to be optimal at the voxel level. However, an area for future evaluation is a more quantitative investigation into the accuracy of results, compared with some kind of "gold standard", possibly defined manually by a group of investigators. In this way fine accuracy could be both optimised and quantified.

An example application of EROS is the pre-alignment of images before registration. Many existing registration methods carry out simple cost-function minimisation over a number of parameters without multiple starts - thus initial positioning often has a great effect on the success of the final registration. An example is the widely used AIR registration program [7,8]. As part of a wider 

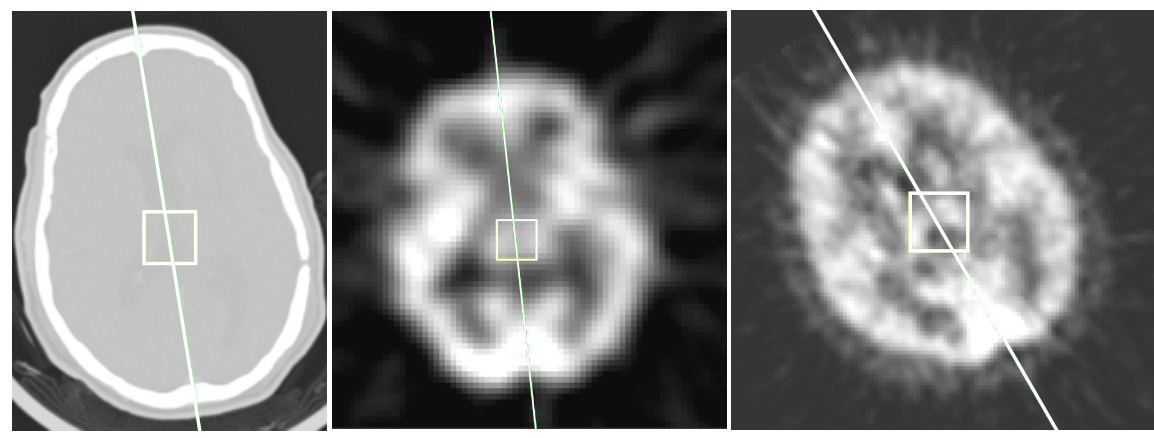

Fig. 4. Slice through 3D CT, SPECT and PET images after symmetry plane detection by EROS.
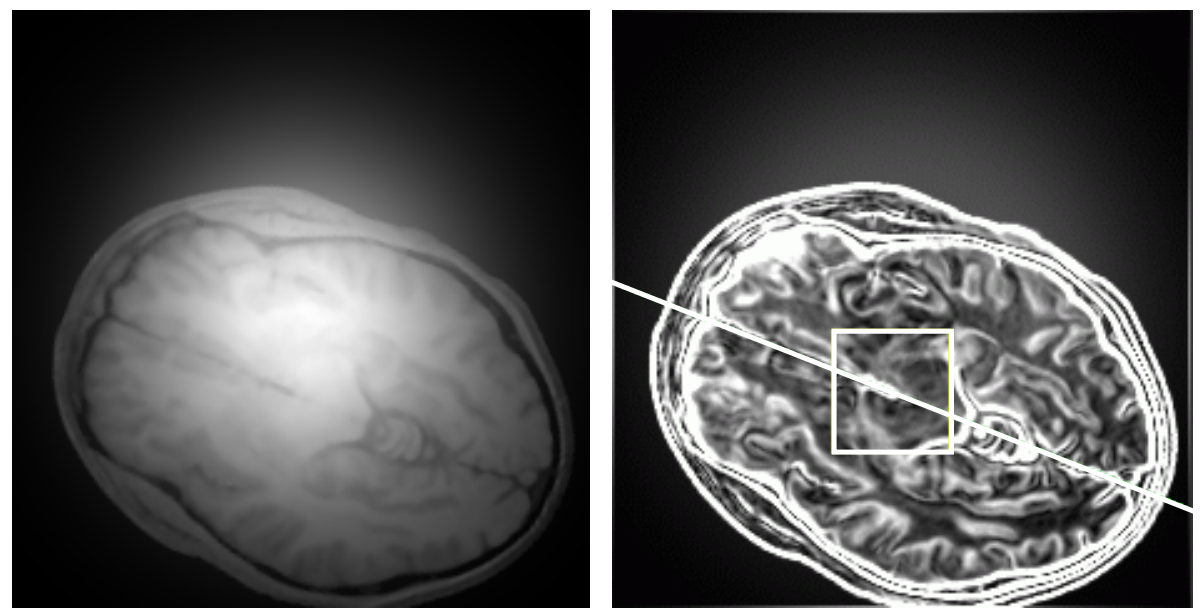

Fig. 5. An image badly corrupted by bias field after symmetry plane detection by EROS with edge enhancement pre-processing.

investigation into registration robustness [2], results from AIR were compared with the results given if EROS was used to pre-align the planes of symmetry from the two images. As Figure 7 shows, for almost all cases, using EROS improved the final registration quality greatly. In fact, EROS correctly aligned the symmetry plane in all cases; where the final registration was not correct after pre-alignment, the error was normally in rotation about the axis perpendicular to the mid-plane, which, obviously, is not at all constrained by finding the symmetry plane. Also, note that EROS is only used to provide a starting estimate to AIR - results could probably be improved if the plane alignment was actually used as a constraint during registration. 

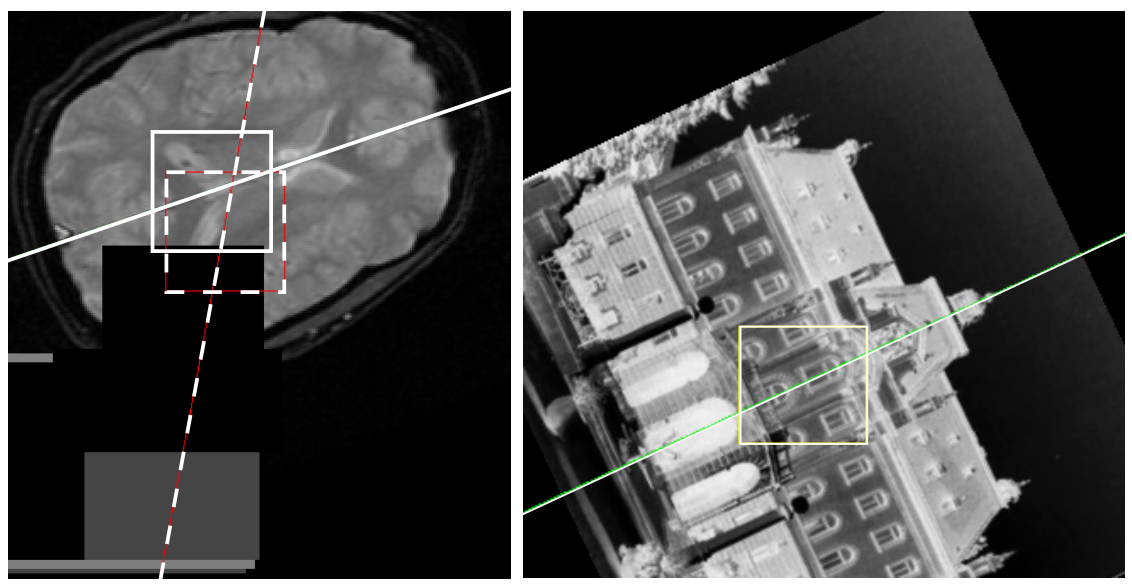

Fig. 6. Slice through 3D MRI (T2-weighted) image after symmetry plane detection by EROS. The image has been artificially corrupted to cause a large asymmetry. Non-robust symmetry detection fails completely (dashed line) whilst robust detection is successful.

Figure 8 shows symmetry detection on two images of rotated shields. In the second case a large amount of noise was added but the result was still good.

An automatic, robust, accurate method has been developed. More work will be carried out to speed it up, and to address the question of rotation about the axis perpendicular to the mid-plane (this is a very different kind of problem). It will be straightforward to extend EROS to allow the estimation of a curved mid- "plane". There is scope for increasing the robustness of EROS by improving the lowest level measure of symmetry, based on means of absoulte values. An alternative should be possible which is robust at even the lowest level to asymmetries, thus increasing even further the power to detect symmetry in objects with asymmetries. It is anticipated that the software will be made freely available from the FMRIB web site within a few months.

\section{References}

1. V. Di Gesù and C. Valenti. Symmetry operators in computer vision. Vistas in Astronomy, 40, 1996. 308

2. M. Jenkinson and S. Smith. Robustness issues in automatic brain image registration. Proc. Int. Conf. on Medical Image Computing and Computer-Assisted Intervention, 1999. submitted. 314

3. S. Minoshima, K.L. Berger, K.S. Lee, and M.A. Mintun. An automated method for rotation correction and centering of three-dimensional functional brain images. Journal of Nuclear Medicine, 33:1579-85, 1992. 309

4. D. Reisfeld, H. Wolfson, and Y. Yeshurun. Context free attentional operators: The generalized symmetry transform. Int. Journal of Computer Vision, 14:119-130, 1995. 308 


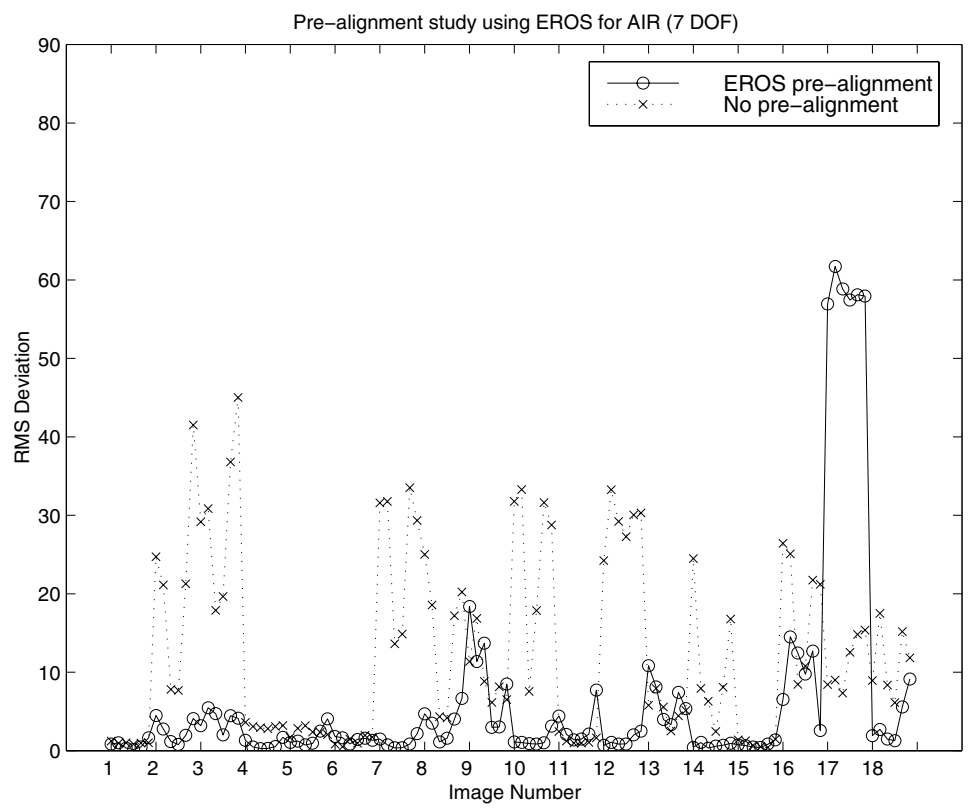

Fig. 7. Application of EROS to pre-alignment for registration - see text for details.
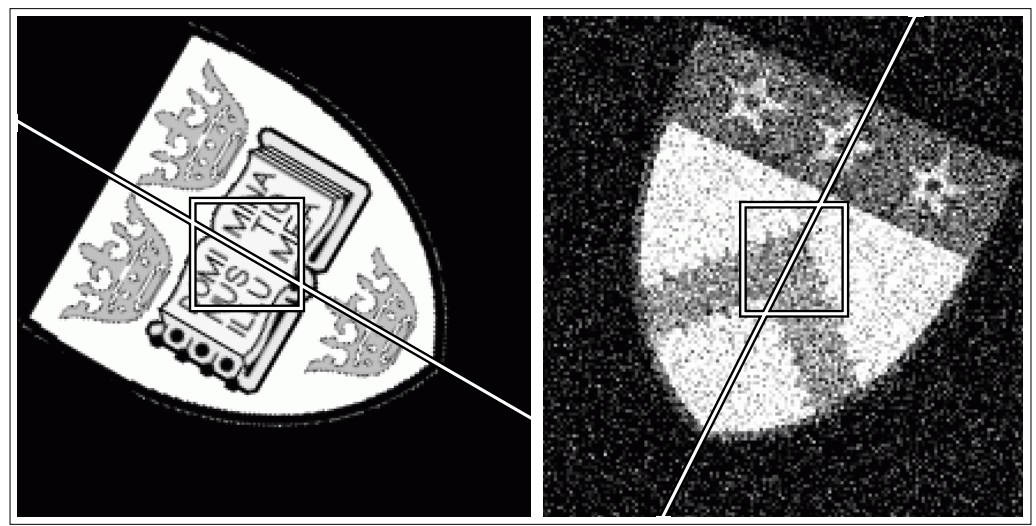

Fig. 8. Images of two shields after symmetry line detection by EROS. The second had Gaussian noise added (maximum image contrast $=255$; noise standard deviation $=50)$. 
5. C. Sun. Symmetry detection using gradient information. Pattern Recognition Letters, 16(9):987-996, 1995. 309

6. J.-P. Thirion, S. Prima, and G. Subsol. Statistical analysis of dissymmetry in volumetric medical images. Technical Report 3178, INRIA Sophia-Antipolis, 1997. 309

7. R.P. Woods, S.R. Cherry, and J.C. Mazziotta. Rapid automated algorithm for aligning and reslicing PET images. Journal of Computer Assisted Tomography, 16(4):620-633, 1992. 313

8. R.P. Woods, J.C. Mazziotta, and S.R. Cherry. MRI-PET registration with automated algorithm. Journal of Computer Assisted Tomography, 17(4):536-546, 1993. 313

9. T. Zielke, M. Brauckmann, and W. von Seelen. Intensity and edge-based symmetry detection applied to car-following. In Proc. 2nd European Conf. on Computer Vision, pages 865-873. Springer-Verlag, 1992. 308 